\title{
Characterization and Evaluation of HepDOCA-doxorubicin Tagged Magnetic Nanoparticles for Hyperthermia
}

Chandraprabha MN*, Lokesh KNL, Rashmi RB, Roshna RG and Vijai SN

Department of Biotechnology, MSRIT, Bangalore, India

\begin{abstract}
This paper reports the hyperemia effect studies of drug-magnetic iron oxide nanoparticle conjugates (MION) for targeted cancer therapy. MIONs were synthesized by the method of co-precipitation using salts of iron as precursors. Chemically modified heparin derivative (heparin deoxycholate, hepDOCA) was complexed to chemotherapeutic drug, doxorubicin (DOX) and tagged to MIONs, which act as the drug-vectors. Each of these conjugates was characterized by SEM and DLS. To observe the effect of formulations, an inductor RF coil circuit was set up which ensured that the MIONs heat up in an AC magnetic field of $0.79 \mathrm{mT}$ at $960 \mathrm{kHz}$. Hyperthermia treatment was performed on E.coli (DH5- $\alpha$ strain) cells and the growth of bacteria along with the protein released upon cell lysis was monitored. It was confirmed that HepDOCA- DOX-MION complex imparts damage to E.coli cells by the magneto-thermo-cytolysis on application of alternating magnetic field.
\end{abstract}

Keywords: MION; Doxorubicin; HepDOCA-DOX-MION conjugate; Hyperthermia; Cells

\section{Introduction}

Conventional cancer therapies employ drugs/radiation that is known to kill cancer cells effectively. However, they risk damage to normal tissues or incomplete eradication of the cancer. Nanotechnology offers the means to aim therapies directly and selectively at cancerous cells. With the advances in polymer nanoparticle technology, the preparation of biodegradable polymeric nanoparticles for drug delivery is gaining significant interest in recent years [1].

Current magnetic nanoparticle technology relies mostly on magnetic iron-oxide nanoparticles (MIONs). The Magnetic fluid Hyperthermia (MFH) is the idea of attaining cytolysis of tumor cells by thermo-cytolysis where the nanoparticles absorb the energy of the magnetic field which is then released into the surrounding area as heat through magnetic "relaxation processes" to produce temperatures between $41^{\circ} \mathrm{C}$ and $45^{\circ} \mathrm{C}$ (hyperthermia), or even higher temperatures between $46^{\circ} \mathrm{C}$ and $70^{\circ} \mathrm{C}$ (thermo ablation) [1]. At temperatures above $46^{\circ} \mathrm{C}$, virtually all biomolecules within the cells are affected, and the tumor cells containing the nanoparticles are directly destroyed as a result of overheating [2].

The transport of drug molecules to specific target sites can be enhanced by the interaction of MIONs with applied magnetic fields while sparing the healthy tissues. Transcellular transport of magnetic iron oxide nanoparticles coated with heparin (Hep-MION) across epithelial cell monolayers on porous polyester membranes have been investigated by Min et al. [3]. Heparin is known to attenuate metastasis by blocking selectin mediated intercellular interactions [4]. Modified derivatives of heparin which can overcome the long term side effects induced by high dose or long-term heparin treatment by the enhanced permeability and retention (EPR) effect have been suggested to be safe drug carriers and as effective drugs for targeted cancer therapy. Doxorubicin (trade name Adriamycin) or hydroxyldaunorubicin is a drug widely used in cancer chemotherapy. Significant progress has been made towards the design and synthesis of polymer-based DOXdelivery systems such as amphiphilic polymeric micelles where DOX is physically encapsulated, and polymer-DOX therapeutics where DOX is chemically encapsulated by covalent binding to polymers [5-7]. Dextran-coated ferric oxide NPs conjugated with specific anti-human epidermal growth factor receptor (HER2) aptamer was used to induce magnetic hyperthermia in cultured cells [8]. Hyperthermia of magnetic nanoparticles (MNPs) has been used to locally and transiently increase blood retina barrier permeability for drug delivery [9]. Deoxycholic acid (DOCA) bearing heparin (HD) amphiphilic conjugates using heparin as a hydrophilic segment and DOCA as a hydrophobic segment have been formulated [10]. In vivo toxicity studies have shown that HD conjugate did not induce unexpected side effects and was safer than free DOX [11-15].

This research was undertaken to focus on the use of magentic iron oxide nanoparticles (MIONs) as drug carriers for heparin-Deoxycholic acid (HepDOCA) conjugate and to demonstrate the thermo-cytotoxic effect by the application of suitable alternating magnetic field.

\section{Expermental}

\section{Method}

N-Hexane from Sigma Aldrich; Heparin, 1-ethyl-3-(3-dimethylaminopropyl) -carbodiimide [EDAC] from Himedia; Doxorubicin. $\mathrm{HCl}$ (Khandelwal Laboratories), Dimethyl Formamide (anhydrous) [DMF], Tetrahydrofuran [THF], Hydroxylamine hydrochloride [HAH], Triethylamine [TEA], Ethylenediamine from SD Fine chemicals; Formamide, $N, N$-dicyclohexylcarbodiimide [DCC] from LOBA Chemie; $N$-Hydroxysuccinimide [NHS] from ROLEX. All reagents used were of ACS grade.

\section{Synthesis of MIONS}

Stock solutions of $1.28 \mathrm{M} \mathrm{FeCl}_{3} \cdot 6 \mathrm{H}_{2} \mathrm{O}, 0.64 \mathrm{M} \mathrm{FeCl}_{2} 4 \mathrm{H}_{2} \mathrm{O}$ were prepared as a source of iron (molar ratio of $\mathrm{Fe}^{3+}: \mathrm{Fe}^{2+}=2: 1$ ) by dissolving

*Corresponding author: Chandraprabha MN, Department of Biotechnology, MSRIT, Bangalore, India, Tel: 919845785174; E-mail: chandraprabhamn@yahoo.com

Received November 23, 2016; Accepted December 22, 2016; Published December 31, 2016

Citation: Chandraprabha MN, Lokesh KNL, Rashmi RB, Roshna RG, Vijai SN (2016) Characterization and Evaluation of HepDOCA-doxorubicin Tagged Magnetic Nanoparticles for Hyperthermia. J Nanomed Nanotechnol 7: 416. doi: 10.4172/2157-7439.1000416

Copyright: ( 2016 Chandraprabha MN, et al. This is an open-access article distributed under the terms of the Creative Commons Attribution License, which permits unrestricted use, distribution, and reproduction in any medium, provided the original author and source are credited. 
Citation: Chandraprabha MN, Lokesh KNL, Rashmi RB, Roshna RG, Vijai SN (2016) Characterization and Evaluation of HepDOCA-doxorubicin Tagged Magnetic Nanoparticles for Hyperthermia. J Nanomed Nanotechnol 7: 416. doi: 10.4172/2157-7439.1000416

Page 2 of 5

the respective chemicals in Milli-Q water under vigorous stirring. Stock solutions of $1 \mathrm{M}, 1.2 \mathrm{M}$ and $1.5 \mathrm{M} \mathrm{NaOH}$ were prepared as alkali sources. Similarly, a solution of $0.01 \mathrm{M} \mathrm{HCl}$ was prepared for surface neutralization. $25 \mathrm{ml}$ of iron source was added drop-wise into $250 \mathrm{ml}$ of alkali source under vigorous mechanical stirring $(2000 \mathrm{rpm})$ for 30 min at room temperature. The $\mathrm{pH}$ conditions were kept constant at 12.8 and MIONs were synthesized for varying concentrations of $1 \mathrm{M}$, $1.2 \mathrm{M}$ and $1.5 \mathrm{M}$ of $\mathrm{NaOH}$. The mixture was gradually heated to $78^{\circ} \mathrm{C}$ and held at this temperature for $1 \mathrm{hr}$ with constant mechanical stirring under $\mathrm{N}_{2}$ protection. The precipitated powder was isolated by applying an external magnetic field, and the supernatant was removed from the precipitate by decantation. Deoxygenated Milli-Q water was added to wash the powder and the solution was decanted after centrifugation at $3500 \mathrm{rpm}$. After washing the powder 2 times, $0.01 \mathrm{M} \mathrm{HCl}$ was added to neutralize the anionic charge on the particle surface. The colloidal suspension was sonicated for 30 mins. The ultrasonicated suspension was filtered through $0.22 \mu \mathrm{m}$ micro filter membrane.

\section{Synthesis of succinimido deoxycholic acid (DOCA)}

DOCA $(11.8$ g, $30 \mathrm{mmol})$ was mixed with $\mathrm{N}, \mathrm{N}$ dicyclohexylcarbodiimide (DCC) $(7.4 \mathrm{~g}, \quad 38.9 \mathrm{mmol})$ and $N$-hydroxysuccinimide (NHS) $(4.5 \mathrm{~g}, 38.2 \mathrm{mmol})$ in $100 \mathrm{mLof}$ tetrahydrofuran (THF). The mixture was reacted for $12 \mathrm{~h}$ at room temperature under nitrogen atmosphere, and then the precipitated dicyclohexylurea was removed by filtration. The filtrate was precipitated in $n$-hexane. The succinimido DOCA precipitate was filtered off and washed thoroughly with $n$-hexane, followed by drying at room temperature. The succinimido DOCA was stored at $-20^{\circ} \mathrm{C}$ before use.

\section{Preparation of DOCA- $\mathrm{NH}_{2}$}

$\mathrm{N}$-Deoxycholyl-ethylenediamine (DOCA- $\left.\mathrm{NH}_{2}\right)$ was synthesized by introducing ethylenediamine to the succinimido DOCA. The succinimido DOCA (1 g, $2 \mathrm{mmol})$ was dissolved in anhydrous $\mathrm{N}, \mathrm{N}$ dimethylformamide (DMF) ( $5 \mathrm{~mL}$ ), and the solution was slowly added dropwise into ethylenediamine $(13.4 \mathrm{~mL}, 0.2 \mathrm{~mol})$ solution. After reaction for $6 \mathrm{~h}$, the mixture was precipitated in distilled water. The white powder DOCA- $\mathrm{NH}_{2}$ was obtained after washing three times with distilled water and was dried.

\section{Preparation of heparin-DOCA (HEPDOCA) conjugates}

$500 \mu \mathrm{l}$ of $167 \mathrm{IU} / \mathrm{mg}$ concentration heparin was mixed with $5 \mathrm{ml}$. of anhydrous formamide by gentle heating. $96 \mathrm{mg}$. of 1-Ethyl-3-(3dimethylaminopropyl)carbodiimide (EDAC) was added to the above heparin solution with $1.086 \mathrm{~g}$. of DOCA- $\mathrm{NH}_{2}$ dissolved in $5 \mathrm{ml}$. of DMF. The resulting solution was stirred at room temperature under nitrogen atmosphere for 24 hours. The mixture was precipitated in excess of cold acetone. The precipitates were carefully washed 3 times with acetone to remove excess DOCA- $\mathrm{NH}_{2}$. The precipitate was then lyophilized and stored at $4^{\circ} \mathrm{C}$.

\section{Preparation of heparin-deoxycholic acid-Doxorubicin (HEPDOCA-DOX) conjugates}

$10 \mathrm{mg}$ of $\mathrm{DOX}-\mathrm{HCl}$ was dissolved in $2 \mathrm{ml}$. of DMF containing triethylamine (TEA) (1.3 mole ratio of DOX-HCl). $100 \mathrm{mg}$. of HD conjugate was dissolved in $5 \mathrm{ml}$. of formamide by gently heating. The 2 solutions were mixed by vortexing. The mixture was ultrafiltered with 10K MWCO membrane to remove all unloaded drugs, DMF, TEA, formamide and triethylammonium chloride (TEA. $\mathrm{HCl}$ ).

\section{Conjugation of heparin deoxycholic-doxorubicin complex to MIONS}

Doxorubicin was entrapped by self-assembled nanoparticles of HepDOCA conjugate by hydrophobic interaction between it and the hydrophobic regions of HepDOCA. $200 \mathrm{~mL}$ of $0.167 \mathrm{mg} \mathrm{Fe} / \mathrm{mL}$ of MION was added to $200 \mathrm{ml}$. of $1 \mathrm{mg} / \mathrm{ml}$. of glycine solution, under stirring condition. The solution was ultrasonicated for $20 \mathrm{mins}$ and was further stirred for 2 hours. $100 \mathrm{ml}$ of $37.5 \mathrm{mg} \mathrm{Fe} / \mathrm{ml}$ of glycine MION was added to $100 \mathrm{ml}$ of HepDOCA-DOX solution under stirring condition and ultrasonicated for 20 mins. Free heparin was removed by ultrafiltration.

\section{Construction of alternating magnetic field circuit for hyperthermia experiments}

Previous research investigations in various fields were considered to construct our magnetic field applicator [12-15]. According to Brown and Neel relaxation, a high frequency magnetic field in terms of close to $\mathrm{MHz}$ range is required to cause the superparamagnetic particles to dissipate heat by continuous fluctuations in the magnetic dipoles. Hence a circuit was developed in order to achieve High frequency alternating magnetic field. The circuit (Figure 1) parameters were as follows:

Coil apparatus: A length of $10 \mathrm{~m}, 21$ SWG thickness was wound over a $4.6 \mathrm{~cm}$ dia plastic cylindrical vessel. About 48 turns were wound
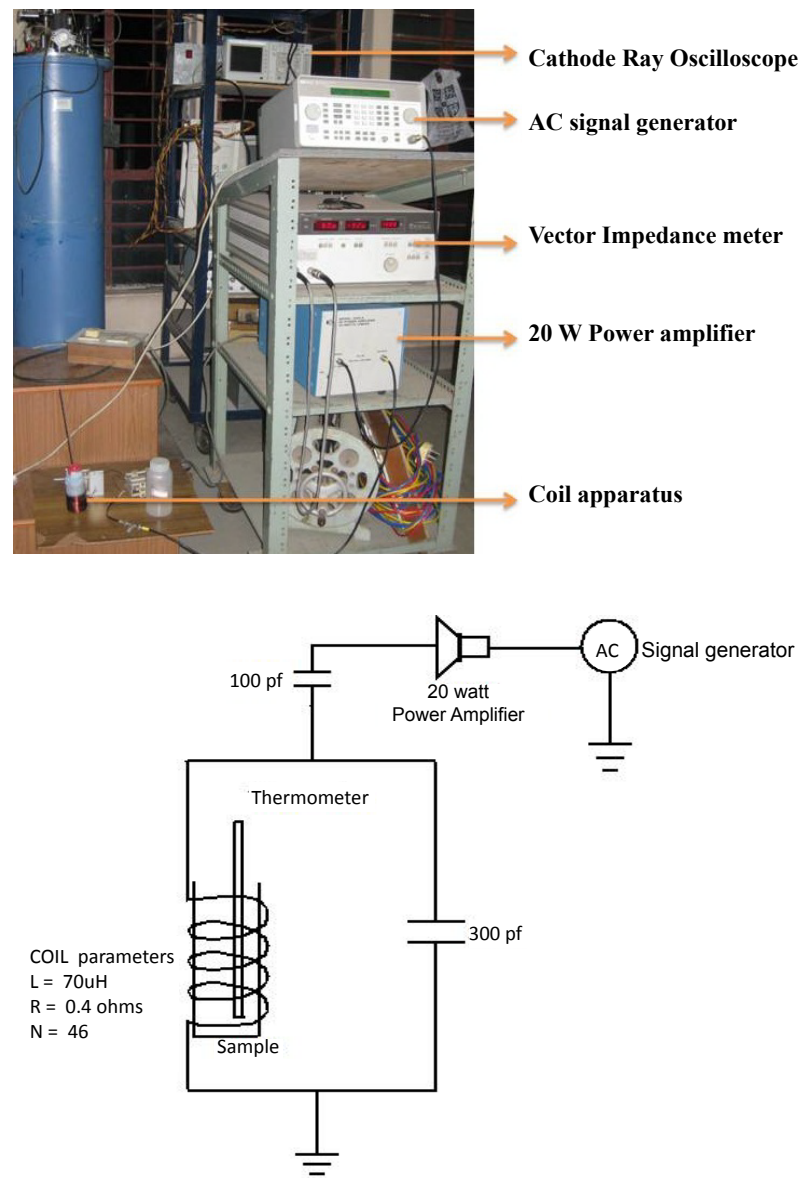

Figure 1: Amplifier circuit for High frequency alternating magnetic field. 
Citation: Chandraprabha MN, Lokesh KNL, Rashmi RB, Roshna RG, Vijai SN (2016) Characterization and Evaluation of HepDOCA-doxorubicin Tagged Magnetic Nanoparticles for Hyperthermia. J Nanomed Nanotechnol 7: 416. doi: 10.4172/2157-7439.1000416

Page 3 of 5

making the solenoid length of $4.6 \mathrm{~cm}$ bearing the values of Inductance, $\mathrm{L}=70 \mu \mathrm{H}$ and Resistance, $\mathrm{R}=0.4 \Omega$, Impedance, $|\mathrm{Z}|=50 \Omega$.

AC signal generator: $960 \mathrm{Khz}$ of signal was supplied from HP 8647A (Range : $250 \mathrm{KHz}-100 \mathrm{MHz}$ )

Power Amplifier: ENI 420LA RF Power Amplifier capable of giving an output of 20 Watts with a gain of $45 \mathrm{~dB}$.

Capacitors: A tuning and matching circuit was designed for the coil based on the frequency. A tuning capacitor of $300 \mathrm{pf}$ (range : 15-490 pf) capacitance and a matching capacitor of 100 pf (range : 3-150 pf) capacitance were used.

Vector Impedance meter: HP 4193A (Range : 0.4-110 MHz) was used to tune the circuit to provide an impedance of $50 \Omega$ at a phase angle approximately $0 \mathrm{deg}$.

Cathode ray oscilloscope: Tektronix TDS 2012B (100 MHz and $1 \mathrm{Gs} / \mathrm{s}$ ).

For the above mentioned parameters, the field strength was calculated to be $0.79 \mathrm{mTesla}$.

\section{Hyperthermia effect on $E$. coli DH5-a cells}

Pure cultures of E. coli DH5- $\alpha$ were prepared by streaking individual colonies on Luria Bertani (LB) agar medium. Single colony was selected and inoculated into Luria Bertani broth and log phase of DH5 - $\alpha$ culture was obtained. The cell count was estimated to be $2 \times 10^{7}$ cells $/ \mathrm{ml}$. The mixtures of cell suspension and the different conjugate formulations at variable concentrations were subjected to alternating magnetic field of strength $0.79 \mathrm{mT}$ at $960 \mathrm{kHz}$. The temperature variation of each of the samples due to the effect of the magnetic field was monitored using an alcohol thermometer. After the heat treatment, the cell suspensions were streaked on to LB agar surface and were analysed for growth characteristics. The amount of protein released from the cells subjected to heat treatment with the various formulations was estimated by Standard Lowry's protein assay and the absorbance at $660 \mathrm{~nm}$ was recorded.

\section{Characterisation studies}

X-ray diffraction studies: The nanoparticles were characterized by X-ray powder diffraction (XRD) with a Philips PW 1830 diffractometer using the monochromatized X-ray beam from the nickel-filtered $\mathrm{Cu}$ Ka radiation.

Scanning electron microscopy studies: Scanning electron microscopy studies were performed by JOEL-JFC 1100E Ion sputtering device.

Dynamic light scattering (DLS): Dynamic light scattering was used to study the size distribution and colloidal stability of drug conjugates. DLS was performed by 90 PLUS - Particle size analyzer (400-700 nm).

\section{Result and Discussion}

\section{Behavior of synthesized MIONS under the action of static magnetic field}

A solution of $30.4 \mathrm{mg} \mathrm{Fe} / \mathrm{ml}$ of MIONs in water was prepared and was subjected to a static field by using a permanent bar magnet. As time proceeded it was observed that, the MION particles agglomerated at the point of application of field (Figure 2) and was steady for long intervals and on removal of the field, they dispersed back into their colloidal form.

\section{X-ray diffraction studied}

The structural properties of $\mathrm{Fe}_{3} \mathrm{O}_{4}$ powders obtained were analyzed by X-ray powder diffraction (XRD) with a diffractometer using the monochromatized $\mathrm{X}$-ray beam from the nickel-filtered $\mathrm{Cu}-\mathrm{Ka}$ radiation. The XRD results (Figure 3 ) indicated crystalline structure with diffraction peaks at $2 \theta$ of $30.2,35.4,43.0,52.7,59.5$ and 63.7, corresponding to indices (220), (311), (400), (422), (511) and (440), respectively of iron oxide. This revealed that the magnetic particles were pure $\mathrm{Fe}_{3} \mathrm{O}_{4}$.

\section{Scanning electron microscope observations}

SEM was performed by JOEL-JFC 1100E Ion sputtering device. The analysis of Scanning electron Microscope photographs (Figure 4) confirmed that the particle size of MIONs and HepDOCA-DOXMION conjugate were well within $200 \mathrm{~nm}$.
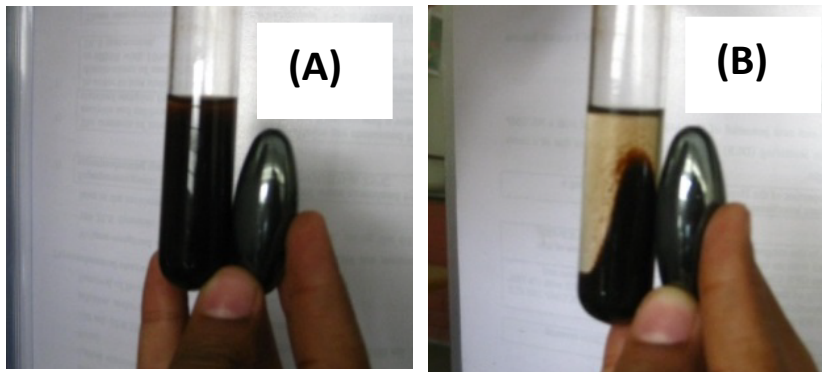

Figure 2: Colloid system of MIONs (A) Before static magnetic field effect and (B) Agglomeration of MIONs after static magnetic field effect.

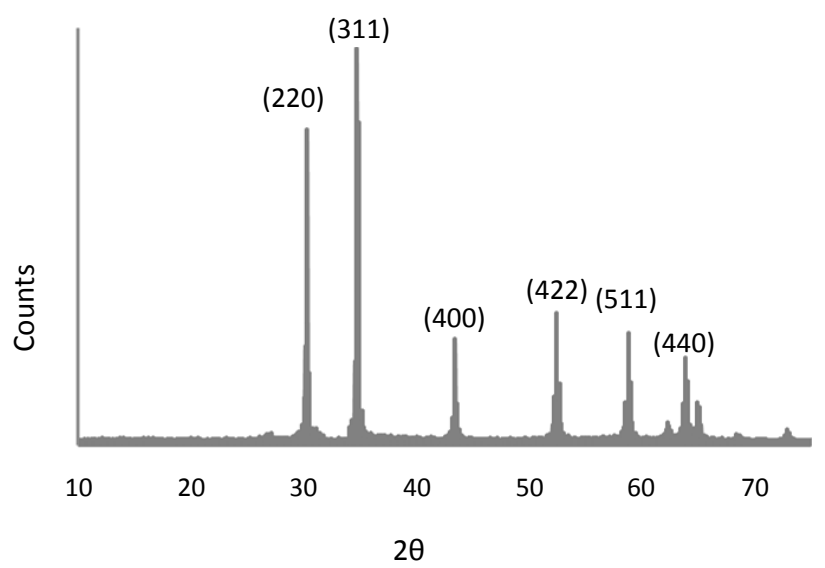

Figure 3: X-ray diffraction of MIONs.

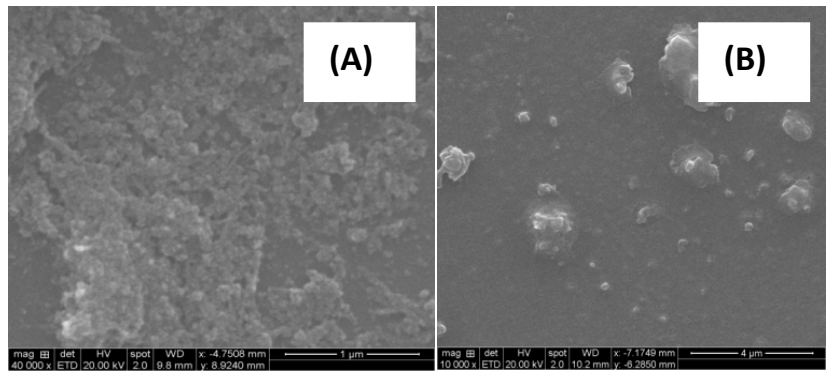

Figure 4: SEM image of (A) MIONs and (B) HepDOCA-DOX-MION conjugate. 
Citation: Chandraprabha MN, Lokesh KNL, Rashmi RB, Roshna RG, Vijai SN (2016) Characterization and Evaluation of HepDOCA-doxorubicin Tagged Magnetic Nanoparticles for Hyperthermia. J Nanomed Nanotechnol 7: 416. doi: 10.4172/2157-7439.1000416

Page 4 of 5

\section{Dynamic light scattering characterization}

DLS was performed by 90 PLUS - Particle size analyzer (400-700 $\mathrm{nm})$ and the results obtained are depicted in Figure 5. The relative intensity values depicted in the graph, generated by the BLC particle sizing software showed that the diameter of HepDOCA particles was in the range of $72.55 \mathrm{~nm}$ to $81.27 \mathrm{~nm}$. The overall mean diameter of HepDOCA was found to be $78.7 \mathrm{~nm}$ as calculated by NNLS algorithm. These results confirm that the conjugates formed were in nanometer size. This assisted us in tagging the HepDOCA conjugate (to Doxorubicin) to maintain the efficacy at nanolevel. The HepDOCADOX particles were in the range of $68.3 \mathrm{~nm}$ to $81.64 \mathrm{~nm}$. The overall mean diameter was found to be $74.21 \mathrm{~nm}$ as calculated by NNLS algorithm. One important aspect to be analyzed is that the mean diameter of HepDOCA-DOX is lesser than that of HepDOCA. This draws an inference that the HepDOCA-DOX conjugate might have stabilized after adding Doxorubicin to HepDOCA which may have cleaved some fragments off HepDOCA leading to its lesser size. The reasons may be due to excessive Doxorubicin concentration or due to higher concentration of HepDOCA used. However the actual causes are yet to be determined.

\section{Temparature response of the different formulations to alternating magnetic field}

The different formulations were subjected to alternating magnetic field of strength $0.79 \mathrm{mT}$ at $960 \mathrm{kHz}$ and the temperature rise in solution was recorded. Care was taken to ensure that the increase in temperature could be attributed in large part to ferromagnetic losses, and not to Ohmic heating from the coil, or infra red absorption. Preliminary results (Figure 6) indicate a constant temperature rise in all samples. A temperature change of about $44^{\circ} \mathrm{C}$ was measured in the suspension of MIONs having $30.4 \mathrm{mg} \mathrm{Fe} / \mathrm{ml}$. The HepDOCA-DOXMION complex had a concentration of $24.5 \mathrm{mg} \mathrm{Fe} / \mathrm{ml}$ and showed the maximum variation in temperature up to $52.2^{\circ} \mathrm{C}$.

\section{Effect of MIONS and HepDOCA-DOX_MION conjugate on cell growth}

The samples subjected to heat treatment by alternating magnetic
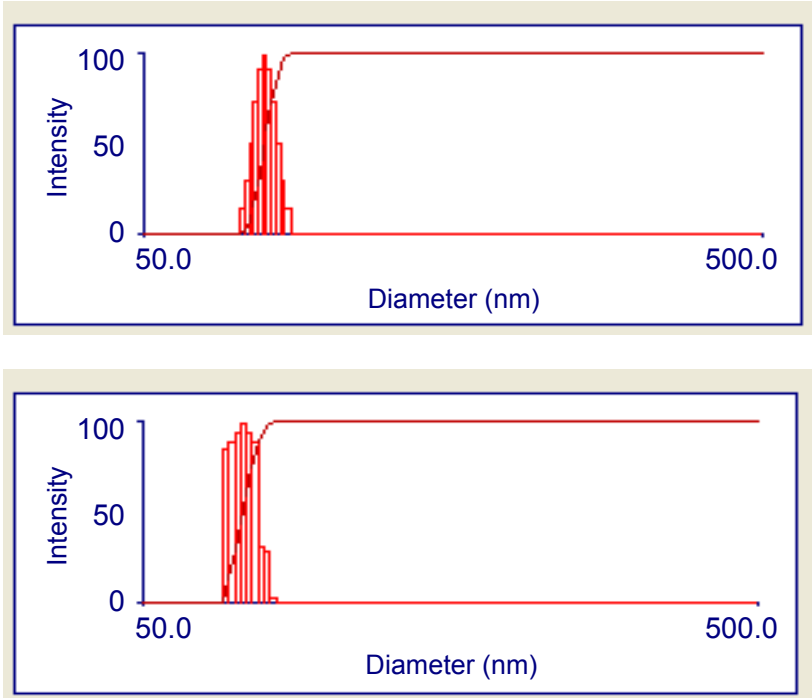

Figure 5: Screen shot showing histogram and mean particle diameter of $(A)$ HepDOCA (B) HepDOCA-DOX conjugate. field as well as those used as control without heat treatment were both plated and incubated for 24 hours to show the growth comparison. It was observed that the colony population of cells interacted with MIONs/HepDOCA-DOX-MION conjugate and subjected to heat treatment was less when compared to control samples without heat treatment (Figure 7). This indicates to the hyperthermia effect of MIONs. The effect was most effective on culture treated with HepDOCA-DOX-MION conjugate formulation indicating the dual activity of the formulation i.e hyperthermia effect of MIONs and the cytotoxic effect of drug.

Figure 8 shows the effect of HepDOCA-DOX-MION complex on

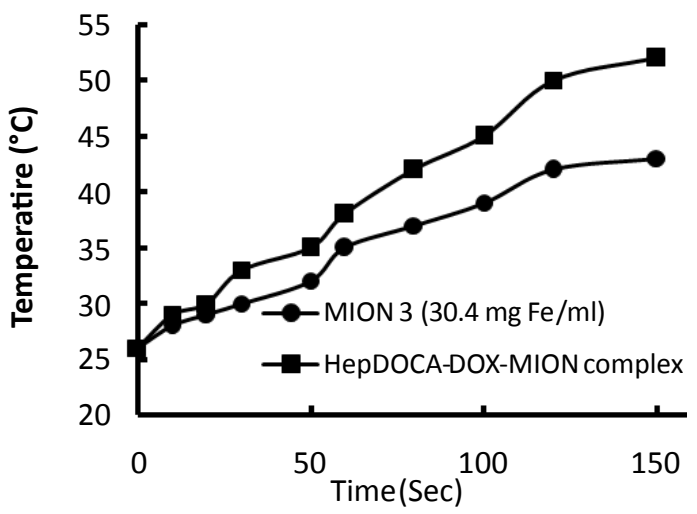

Figure 6: Temperature variations of different formulations with cell culture under the action of magnetic field.
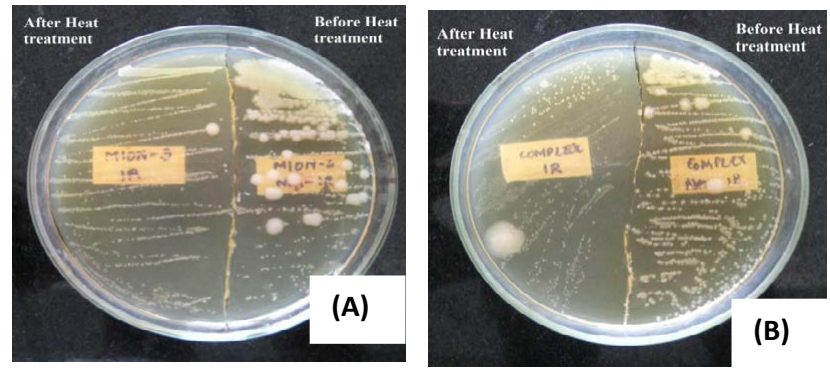

Figure 7: Cell colony density comparison for cells treated with (A) MIONs (B) HepDOCA-DOX-MION conjugate.

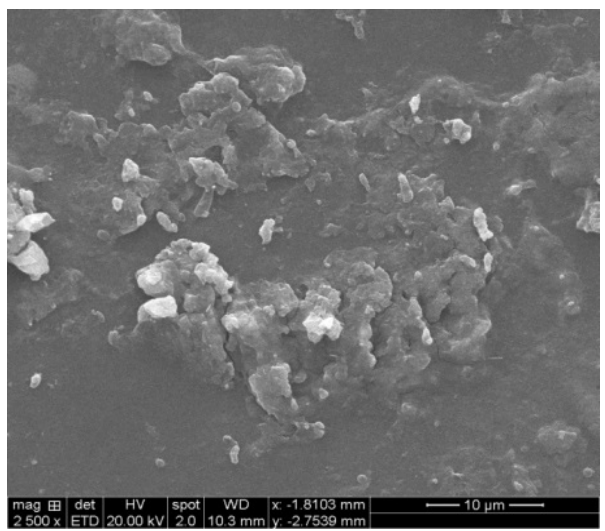

Figure 8: SEM image of cells treated with HepDOCA-DOX-MION showing agglomeration and cell lysis. 
Citation: Chandraprabha MN, Lokesh KNL, Rashmi RB, Roshna RG, Vijai SN (2016) Characterization and Evaluation of HepDOCA-doxorubicin Tagged Magnetic Nanoparticles for Hyperthermia. J Nanomed Nanotechnol 7: 416. doi: 10.4172/2157-7439.1000416

cells as seen under SEM after treatment with alternating magnetic field. The image shows agglomeration of lysed cells substantiating the effect of the complex.

\section{Estimatioin of protein content released by cells}

Cells interacted with various formulations were subjected to heat treatment and the protein content released was estimated. Protein released was found to be $2.1 \mathrm{mg} / \mathrm{ml}$ for the control E. coli cells, 5.0 $\mathrm{mg} / \mathrm{ml}$ for cells treated with $30.4 \mathrm{mg} \mathrm{Fe} / \mathrm{ml} \mathrm{MION}$ and $9.6 \mathrm{mg} / \mathrm{ml}$ for cells treated with HepDOCA-DOX-MION complex. The higher concentration of protein released indicates the extent of cell damage caused by the thermal effects. The highest protein release was observed on adding of HepDOCA-DOX-MION complex which might account for the action of both the drugs (heparin deoxycholate and doxorubicin) along with hyperthermia effect.

\section{Conclusion}

A unique approach to tag the MIONs to two drug molecules (Heparin deoxycholic acid and Doxorubicin) to obtain HepDOCADOX-MION complex was established. Magnetic heating apparatus was set up to ensure that the MIONs heat up in an AC magnetic field of 0.79 $\mathrm{mT}$ at $960 \mathrm{kHz}$. Different characterization techniques were pursued to characterize the newly fabricated particles. The overall mean diameter of HepDOCA and HepDOCA-DOX determined by dynamic light scattering was found to be $78.7 \mathrm{~nm}$ and $74.21 \mathrm{~nm}$ respectively. These results confirm that the conjugates formed were in nanometer size. SEM images provided additional evidence to prove that the particles were in nano size range. The colony population of cells interacted with MIONs/HepDOCA-DOX-MION conjugate and subjected to heat treatment was significantly less compared to control samples without heat treatment indicating the detrimental effect of hyperthermia. Protein release from cells interacted with HepDOCA-DOX-MION complex was higher than for cells treated with only MION's accounting for the dual action of the drugs (heparin deoxycholate and doxorubicin) along with hyperthermia effect.

\section{References}

1. Perez CA, Emami B, Myerson RJ (1992) Hyperthermia, In Principles and practice of radiation oncology. Lippincott, Philadelphia 396-446.
2. Oleson JR, Calderwood SK, Coughlin CT (1988) Biological and clinical aspects of hyperthermia in cancer therapy. American Journal of Clinical Oncology 11: 368-380.

3. Min KA, Yu F, Yang VC, Zhang X, Gus R (2010) Transcellular transport of heparin-coated magnetic iron oxide nanoparticles. (hep-mion) under the influence of an applied magnetic field. Pharmaceutics Journal 2: 119-135.

4. Park K, Kim YS, Lee GY, Nam JO, Lee SK, et al. (2007) Antiangiogenic effect of bile acid acylated heparin derivative. Pharmaceutical Research 24: 176-185.

5. Shi M, Ho K, Keating A, Shoichet MS (2009) Doxorubicin-conjugated immunonanoparticles for intracellular anticancer drug delivery. Advanced Functional Materials 11: 1689-1696.

6. Min KA, Lee HJ, Kim K, Kwon IC, Jeong SY, et al. (2012) The tumor accumulation and therapeutic efficacy of doxorubicin carried in calcium phosphate-reinforced polymer nanoparticles. Biomaterials 33: $5788-5797$.

7. Amanda LG, James BB, Jeremy SP, Sarah MN, David EN, et al. (2013) Magnetic heating of iron oxide nanoparticles and magnetic micelles for cancer therapy. IEEE Trans Magn 49: 231-235.

8. Katarzyna P, Anna S, Filip J, Piotr J, Jacek O (2014) Tumor-specific hyperthermia with aptamer-tagged superparamagnetic nanoparticles. International Journal of Nanomedicine 9: 67-76.

9. Seyed NT, Maryam ST, Hélène G, Sylvain M (2016) Hyperthermia of magnetic nanoparticles allows passage of sodium fluorescein and Evans blue dye across the blood-retinal barrier. International Journal of Hyperthermia 32: 657-665.

10. Park K, Kim K, Kwon IC, Kim SK, Lee S, et al. (2004) Preparation and characterization of self-assembled nanoparticles of heparin-deoxycholic acid conjugates. Langmuir journal 20: 11726-11731.

11. Park K, Kim K, Kwon IC, Kim SK, Lee S, et al. (2006) Heparin-deoxycholic acid chemical conjugate as an anticancer drug carrier and its antitumor activity. Journal of controlled release 111: 228-234.

12. Kalambur VS, Han B, Hammer BE, Shield TW, Bischof JC (2005) In vitro characterization of movement, heating and visualization of magnetic nanoparticles for biomedical applications. Nanotechnology 16: 1221.

13. Hergt R, Dutz S, Muller R and Zeisberger M (2006) Magnetic particle hyperthermia: nanoparticle magnetism and materials development for cancer therapy. Journal of Physics: Condensed Matter 18: 2919-2934.

14. Pankhurst QA, Connolly J, Jones SK, Dobson J (2003) Applications of magnetic nanoparticles in biomedicine. Journal of Physics D: Applied Physics 36: 167-181.

15. Iddo M, Gescheit, David MB, Gannot I (2008) A proposed method for thermal specific bioimaging and therapy technique for diagnosis and treatment of malignant tumors by using magnetic nanoparticles. Advances in Optical Technologies. 